\title{
D'un producteur clandestin de drogue à la maladie de Parkinson
}

Les nouvelles

de ce numéro

ont été rédigées par :

Jean-Claude Dreyfus

Axel Kahn

Jean-Pierre Grünfeld

(I) Par exemple, quatre articles sont parus dans Science et trois dans Nature au cours des six derniers mois de 1984 .

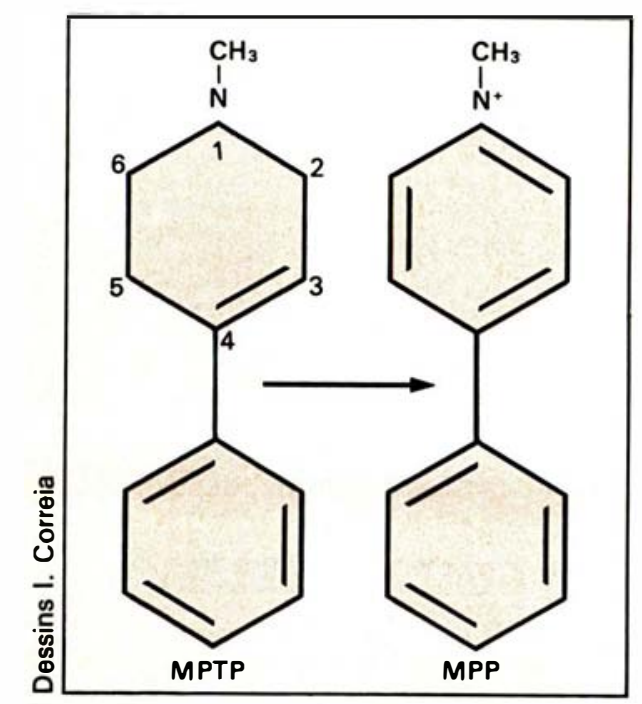

Oxydation de MPTP en MPP ${ }^{+}$

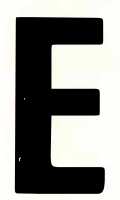

n 1979, paraît dans une revue intitulée Psychiatry Research un article refusé par plusieurs journaux. Il relate l'histoire d'un étudiant de 23 ans qui, en 1976, voit se développer un syndrome parkinsonien après absorption d'une drogue synthétisée par lui-même. Par suite de simplifications techniques, sa préparation contenait un contaminant : la I-méthyl-4-phényl tétrahydropyridine (MPTP). Il meurt d'un surdosage en 1978, et l'autopsie révèle que les cellules de son locus niger sont très raréfiées. En 1982, un homme de 42 ans est admis à l'hôpital de San José, incapable de parler et de se mouvoir. Une semaine plus tard, sa sœur est admise à son tour. Ils avaient tous deux acheté une "héroïne de synthèse " à un vendeur de rue. L'analyse du produit toxique est en cours lorsque le rapprochement est fait entre les deux histoires; et le produit actif est alors identifié : c'est le MPTP.

Les chercheurs partent alors sur les traces du MPTP, synthétisé pour la première fois en 1947. En raison d'une similitude de formule avec certains neurotransmetteurs, on décide de l'essayer comme antiparkinsonien. Les résultats initiaux conduisent à abandonner ce traitement, littéralement homéopathique. La partie expérimentale du travail n'aboutit pas, probablement parce que les rats ne se montrent pas sensibles au MPTP. Pourtant, deux macaques Rhesus présentent une rigidité progressive qui échappe à l'attention des investigateurs. Dès que les chercheurs de 1982 retrouvent ces informations dans les archives, ils reprennent en détail l'effet du MPTP sur les singes, et les publications affluent (I).

Ces travaux ont réveillé l'intérêt pour les mécanismes de base de la maladie de Parkinson, quelque peu négligés depuis la découverte de l'activité thérapeutique de la L-dopa. On sait que la maladie comporte des destructions cellulaires dans la substance noire mais aussi dans d'autres zones comme le locus coeruleus. Les cellules émettent des prolongements capables de capter la dopamine à partir du corps strié, et leur carence bloque la fonction motrice, sans que le mécanisme de ce déficit soit encore correctement élucidé. Le tableau dû au MPTP, cliniquement très voisin, n'atteint anatomiquement que le locus niger, ce qui conduit à y localiser la lésion primitive. Dans l'organisme le MPTP est rapidement oxydé en I-méthyl-4-phényl pyridine (MPP). Le cerveau possède des récepteurs qui sont capables de fixer le MPTP. La densité de ces récepteurs serait plus grande que chez le rat pour l'homme, certains singes et la souris. L'oxydation en MPP est catalysée par la mono-aminoxydase et plus particulièrement son isozyme B. D'après des études comparatives en autoradiographie, le récepteur au MPTP pourrait être la MAO de type B elle-même. Les inhibiteurs de la MAO B, pargyline et surtout deprenil, bloquent l'oxydation et empêchent l'apparition des symptômes de l'intoxication expérimentale. Ces observations ont d'ailleurs ravivé l'intérêt pour les inhibiteurs de la MAO aux États-Unis où ces médicaments n'avaient guère été utilisés.

Les recherches expérimentales précitées ont permis de dégager l'hypothèse d'une origine toxique possible de la maladie de Parkinson. Certes, des différences notables subsistent entre la maladie clinique et l'intoxication au MPTP. Il n'est toutefois pas surprenant qu'une atteinte aiguë ne rende pas compte de l'évolution d'une affection qui s'étale sur des années. Le modèle du macaque présente du moins l'avantage de se prêter à ce type d'étude, en donnant de faibles doses pendant un temps prolongé. Il ne s'agit là encore que d'hypothèses. Reste à savoir pourquoi les cellules du locus niger sont affectées spécifiquement, et si le MPTP ou tout autre toxique joue effectivement un rôle dans la maladie humaine. 